\title{
Effect of adrenocorticotropic hormone infusion on circulating sclerostin levels
}

\author{
Sarah Zaheer ${ }^{1}$, Kayla Meyer ${ }^{2}$, Rebecca Easly ${ }^{2}$, Omar Bayomy ${ }^{3}$, Janet Leung ${ }^{4}$, Andrew W Koefoed ${ }^{2}$, \\ Mahyar Heydarpour', Roy Freeman ${ }^{5,6}$ and Gail K Adler ${ }^{2,3,5}$ \\ 'Division of Endocrinology, Metabolism, and Nutrition, Duke University Medical Center, Durham, North Carolina, USA \\ 2Division of Endocrinology, Diabetes and Hypertension, Brigham and Women's Hospital, Boston, Massachusetts, USA \\ ${ }^{3}$ Department of Medicine, Brigham and Women's Hospital, Boston, Massachusetts, USA \\ ${ }^{4}$ Section of Endocrinology, Virginia Mason Medical Center, Seattle, Washington, USA \\ ${ }^{5}$ Harvard Medical School, Boston, Massachusetts, USA \\ ${ }^{6}$ Department of Neurology, Beth Israel Deaconess Medical Center, Boston, Massachusetts, USA \\ Correspondence should be addressed to G K Adler: gadler@bwh.harvard.edu
}

\begin{abstract}
Glucocorticoid use is the most common cause of secondary osteoporosis. Poor skeletal health related to glucocorticoid use is thought to involve inhibition of the $\mathrm{Wnt} / \beta$-catenin signaling pathway, a key pathway in osteoblastogenesis. Sclerostin, a peptide produced primarily by osteocytes, is an antagonist of the $\mathrm{Wnt} / \beta$-catenin signaling pathway, raising the possibility that sclerostin is involved in glucocorticoids' adverse effects on bone. The aim of this study was to determine whether an acute infusion of cosyntropin (i.e. ACTH(1-24)), which increases endogenous cortisol, increases serum sclerostin levels as compared to a placebo infusion. This study was performed using blood samples obtained from a previously published, double-blind, placebo-controlled, randomized, cross-over study among healthy men and women who received infusions of placebo or cosyntropin after being supine and fasted overnight (ClinicalTrials.gov NCT02339506). A total of 17 participants were analyzed. There was a strong correlation $\left(R^{2}=0.65, P<0.0001\right)$ between the two baseline sclerostin measurements measured at the start of each visit, and men had a significantly higher average baseline sclerostin compared to women. As anticipated, cosyntropin significantly increased serum cortisol levels, whereas cortisol levels fell during placebo infusion, consistent with the diurnal variation in cortisol. There was no significant effect of cosyntropin as compared to placebo infusions on serum sclerostin over 6-24 $\mathrm{h}$ $(P=0.10)$. In conclusion, this randomized, placebo-controlled study was unable to detect a significant effect of a cosyntropin infusion on serum sclerostin levels in healthy men and women.
\end{abstract}
Key Words
- sclerostin
- $\mathrm{ACTH}$
- cortisol
- glucocorticoid
- bone

\section{Introduction}

Sclerostin, an anti-anabolic glycoprotein produced primarily by osteocytes, is an antagonist of the Wnt/ $\beta$-catenin signaling pathway, the principle pathway in osteoblastogenesis $(1,2,3$, $4,5,6,7,8,9)$. Glucocorticoids also have a negative impact on the Wnt/ $\beta$-catenin pathway leading to an imbalance in bone formation and bone resorption, and thereby contributing to glucocorticoid-induced osteoporosis $(1,3,10,11,12$, 13). In mice, glucocorticoids increase the expression of sclerostin (14, 15). Administration of a sclerostin-neutralizing antibody or knockout of Sost, the gene encoding sclerostin, reduces glucocorticoid-induced bone loss in mice $(16,17,18,19)$. Thus, the adverse effect of glucocorticoids on bone formation may be mediated, in part, by stimulation of the production of sclerostin.

The impact of glucocorticoids on circulating sclerostin levels in humans is uncertain. Increases and decreases 
in serum sclerostin have been reported in patients with endogenous hypercortisolemia and in patients receiving exogenous glucocorticoids for underlying medical conditions $(20,21,22,23,24,25,26,27)$. To our knowledge, there is only one randomized study examining the effect of glucocorticoids vs placebo on sclerostin. This study demonstrated a significant decrease in serum sclerostin with a high (30 mg/day), but not low ( $7.5 \mathrm{mg} /$ day), dose of prednisolone given over 2 weeks in healthy young men (28). These studies examined the effect of glucocorticoids on sclerostin over weeks to months.

Other studies have demonstrated that sclerostin levels can be acutely regulated over minutes to hours in humans. Serum sclerostin levels are increased immediately following a bout of intense physical exercise in healthy humans $(29,30,31)$. This increase in sclerostin is accompanied by activation of the pituitary-adrenal axis, raising the possibility that acute increases in cortisol due to pituitaryadrenal activation increase sclerostin.

Therefore, the goal of this study was to determine if an infusion of cosyntropin (i.e. adrenocorticotropin hormone(1-24) $(\mathrm{ACTH}(1-24)))$, as compared to placebo, increases serum sclerostin levels in healthy men and women within $24 \mathrm{~h}$.

\section{Methods}

We performed a secondary analysis using blood samples obtained from a previously published, double-blind, placebo-controlled, randomized, cross-over study among healthy individuals who received an infusion of placebo or ACTH (ClinicalTrials.gov NCT02339506) (32). The primary aim in the published study was to assess the role of the ACTH-adrenal axis in decreasing baroreflex sensitivity in healthy individuals. This is a biochemical sub-analysis. Participants were between 18 and 55 years of age with BMI of $18-32 \mathrm{~kg} / \mathrm{m}^{2}$. Exclusion criteria included any evidence of active medical illnesses based on history, physical examination, ECG, and screening blood tests. In addition, subjects were excluded for pregnancy and/or prescription or herbal medication use, including steroid use within the past 6 months, with the exception of oral contraceptives. The study protocol was performed at the Brigham and Women's Hospital Center for Clinical Investigation (Boston, MA, USA). The Partners Healthcare Institutional Review Board approved the protocol. Informed consent was obtained from all individual participants included in the study. Sample size was based on our published study (32).

\section{Center for clinical investigation study visits}

Participants completed two 3-day/2-night in-patient study visits at the Center for Clinical Investigation. On one visit, participants received cosyntropin and on the other placebo, and the visits were separated by a 1-3 months wash-out period (Fig. 1). Participants were randomized to treatment group (placebo then cosyntropin, or cosyntropin then placebo) with equal probability using a permutated block method. Randomization was 1:1. All participants, study staff, and nursing staff were blinded to the treatment. The randomization codes were generated by a biostatistician and provided in sealed envelopes to the Brigham and Women's Hospital investigational pharmacy. The research pharmacists did not have any contact with the research participants and did not communicate assigned treatments with any of the study staff. Study staff were only unblinded to assigned treatments for data analysis at the conclusion of the study. One week prior to each study visit, subjects were asked to abstain from alcohol, caffeine, and excessive exercise, as well as to consume their normal diet with an additional two bouillon packets per day to standardize sodium intake. Participants were then admitted to the research center for their 3-day admission and remained on fasting and supine after midnight.

The next morning, participants received two 2.5-h infusions (one infusion starting at approximately 09:30 $\mathrm{h}$ and one starting at approximately 13:30 h) of either cosyntropin (ACTH(1-24), $70 \mu \mathrm{g} / \mathrm{h}$, Sandoz Pharmaceuticals) in both, the morning and afternoon or $0.9 \%$ normal saline in both the morning and afternoon. Participants received the alternate infusion for their second admission. On each visit, the two infusions were separated by a 90-min break during which participants were allowed to sit upright for $30 \mathrm{~min}$ and consume a small breakfast. Subjects were otherwise asked to remain supine for the duration of the infusions. At the conclusion of the second infusion, participants were allowed to eat and ambulate until midnight ad libitum, when they were asked again to fast and remain supine until the conclusion of the study procedures the following morning on Study Day 2 . After morning evaluations on Study Day 2, participants were discharged.

During all study visits, an intravenous line was placed retrograde into a hand or wrist vein to withdraw blood samples. This line was placed at least an hour before blood samples were drawn. Blood samples obtained at baseline, at the end of the second infusion, and the morning of Study Day 2 ( $24 \mathrm{~h}$ after the baseline measure) were assayed for sclerostin (Fig. 1). In addition, serum

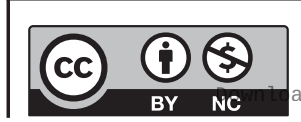

This work is licensed under a Creative Commons Attribution-NonCommercial 4.0 International License. ded from Bioscientifica.com at 04/26/2023 04:54:19AM 
Placebo Study Visit

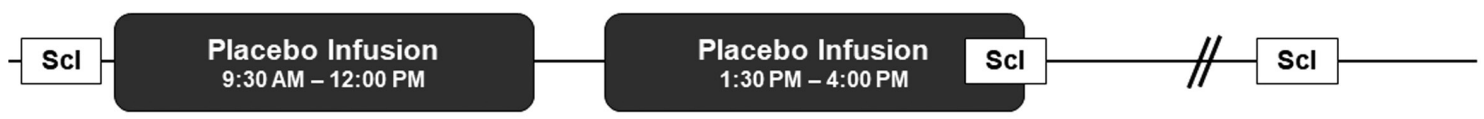

\section{Cosyntropin Study Visit}

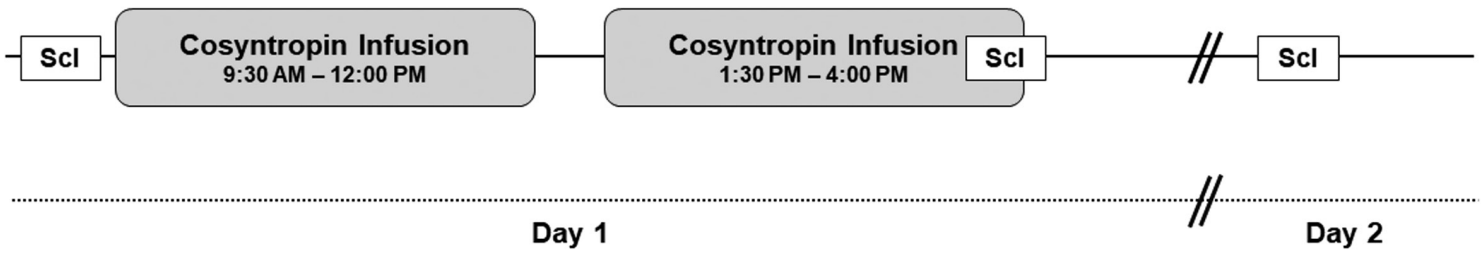

Figure 1

Inpatient study design. Participants completed two study visits in random order with a 1-3 months wash-out period between the visits. At one study visit, they received two $2.5 \mathrm{~h}$ infusions of placebo ( $0.9 \%$ saline) and at the other visit they received two $2.5 \mathrm{~h}$ infusions of synthetic $\mathrm{ACTH}(1-24)$ (cosyntropin) $70 \mu \mathrm{g} / \mathrm{h}$. Scl refers to the assessment of sclerostin levels. These were taken at baseline (before the morning infusion), immediately preceding the end of the second infusion, and the following day ( $24 \mathrm{~h}$ after baseline measures). Sclerostin levels were evaluated for both arms of the study and at both visits for each participant. Study design diagram is adapted from our previously published study (Leung et al. 2020).

cortisol was assessed at baseline, immediately prior to the start of each infusion, at 30-45 min intervals during the infusions, and the next morning. Twenty-four-hour urine collections were obtained beginning the morning of the infusion (Study Day 1) and ending the following morning (Study Day 2) and assayed for urinary-free cortisol and creatinine.

\section{Laboratory analysis}

Sclerostin levels were measured in plasma EDTA samples using the Quantikine Human SOST Immunoassay (R\&D Systems) by the Research Laboratory Services at Maine Medical Center (Portland, ME, USA). Sensitivity of this assay is $3.8 \mathrm{pg} / \mathrm{mL}$, and the assay range is 31.30-2000 pg/mL. Serum cortisol was measured using standard clinical laboratory improvement amendments (CLIA) certified laboratory techniques (Beckman Coulter, Inc, Brea, CA, USA), and urinary-free cortisol was measured using mass spectrometry at the Brigham Research Assay Core. Urine creatinine was measured at a CLIA certified laboratory (LabCorp, Burlington, NC, USA).

\section{Statistical analysis}

The primary outcome for the current analysis was the effect of cosyntropin on circulating serum sclerostin levels. Secondary outcomes included analyzing the 24-h change in serum sclerostin in men and women separately and assessing whether age, sex, BMI, or urine-free cortisol were predictors of serum sclerostin levels. Data are expressed in mean \pm S.D. for normally distributed data and as median (interquartile range) for non-normally distributed data. Serum sclerostin data were not normally distributed at all time points for both placebo and cosyntropin infusions. Therefore, data were log-transformed prior to analysis. We used a mixed model for the analysis, adjusting for fixed factors (age, sex, time, and BMI) and random factor (each individual), to compare cosyntropin treatment vs placebo treatment over time. Non-repeated measures assessed in two conditions were analyzed using Student's two-tailed $t$-test. For comparisons of baseline sclerostin, data were logtransformed prior to performing the Student's two-tailed $t$-test. Linear regression models were used to assess the relationships between the variables. Categorical variables were compared using Fisher's exact test. $P$ values $<0.05$ were considered significant. Statistical analysis was performed with JMP ${ }^{\circ}$, Version < 12 pro>. SAS Institute Inc., Cary, NC, USA 1989-2019.

\section{Results}

\section{Study participants and clinical characteristics}

A total of 18 healthy participants were randomized and completed both the cosyntropin and placebo study visits.

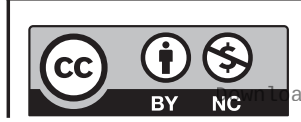


Of these, 17 participants had serum samples available for determination of sclerostin levels and were included in the analysis. The mean age of the participants was $32 \pm 13$ years with mean BMI of $23.6 \pm 3.3 \mathrm{~kg} / \mathrm{m}^{2}$. In the study, 10 out of 17 participants were male and 12 out of 17 were Caucasian. Of the seven female participants, two were taking oral contraceptive pills. Baseline characteristics and demographics are shown in Table 1. Recruitment and follow-up occurred during the period lasting from March 2015 to March 2016. There were no significant harms or unintended effects observed with either treatment.

\section{Association of sclerostin with baseline characteristics}

We measured the baseline sclerostin levels at the start of each study visit, which occurred $1.4 \pm 0.7$ months apart. An interaction effect model showed no carry-over effect of treatment on sclerostin $(P=0.995)$. There was a strong correlation $\left(\mathrm{R}^{2}=0.65, P<0.0001\right)$ between the two baseline sclerostin measurements measured at the start of each visit, demonstrating stability in sclerostin levels over time within an individual (Fig. 2). The average baseline sclerostin level for each participant was determined by averaging the baseline measurements from study visit 1 to study visit 2 . There was a wide range of average baseline sclerostin values between individuals, from 228.5 to $782.9 \mathrm{pg} / \mathrm{mL}$ (range $554.4 \mathrm{pg} / \mathrm{mL}$ ). Men had a significantly higher average baseline sclerostin compared to women (533.0 (389.8-561.0) pg/mL vs 249.5 (245.4-323.9) pg/mL, $P=0.001)$. Seventy percent of the men had baseline sclerostin values higher than the maximum value observed in women. Using a linear regression model, sex was a predictor of baseline sclerostin $\left(R^{2}=0.46\right)$, whereas age and BMI did not predict baseline sclerostin levels $\left(\mathrm{R}^{2}<0.01\right.$ and $\mathrm{R}^{2}<0.0001$, respectively).

Table 1 Participant demographics and screening characteristics $(n=17)$. Data are expressed as percentage or mean \pm S.D.

\begin{tabular}{|c|c|}
\hline Parameter & Value \\
\hline Age (years) & $32 \pm 13$ \\
\hline Sex & $41 \%$ Female, 59\% Male \\
\hline Race (self-identified) & $\begin{array}{l}6 \% \text { Asian, } 23 \% \text { African } \\
\text { American or Black, } 71 \% \text { White }\end{array}$ \\
\hline Latino or Hispanic & $6 \%$ \\
\hline $\mathrm{BMI}\left(\mathrm{kg} / \mathrm{m}^{2}\right)$ & $23.6 \pm 3.3$ \\
\hline Systolic blood pressure (mmHg) & $116 \pm 9$ \\
\hline Diastolic blood pressure (mmHg) & $70 \pm 8$ \\
\hline Heart rate (b.p.m.) & $79 \pm 11$ \\
\hline
\end{tabular}

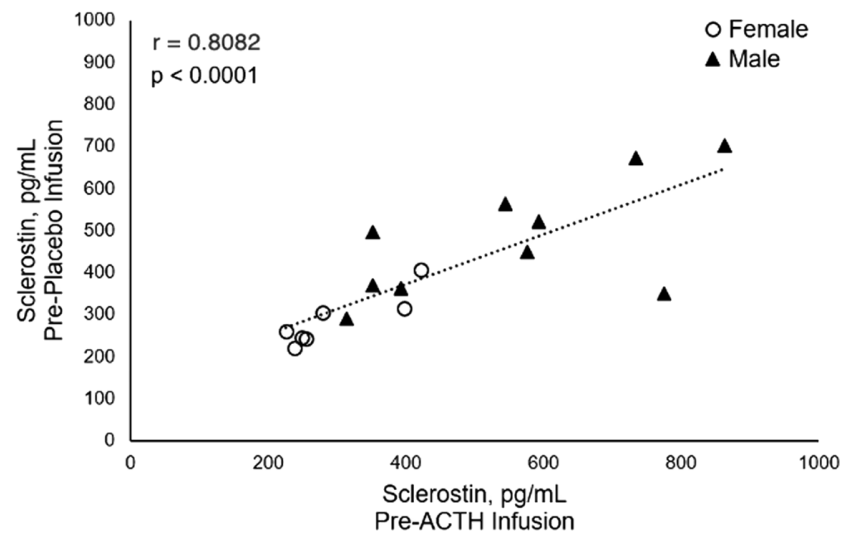

Figure 2

Baseline sclerostin correlated by visit. Bivariate correlation between each individual's baseline sclerostin values at the placebo visit and cosyntropin visit. Baseline sclerostin measurements were significantly correlated, $P<0.0001$ (linear regression). There was a wide range of baseline sclerostin levels, however, for a given individual it remained consistent over the 1-3 months wash-out period. Open circles denote female participants and solid triangles denote male participants.

\section{Impact of cosyntropin infusion on cortisol}

Cosyntropin infusion significantly increased serum cortisol levels, from $11.8 \pm 4.4 \mu \mathrm{g} / \mathrm{dL}$ at baseline to $37.0 \pm 5.9$ $\mu \mathrm{g} / \mathrm{dL}(P<0.0001)$ at the end of the afternoon infusion. In comparison, serum cortisol significantly decreased throughout the day during the placebo infusion, dropping from $11.2 \pm 3.8$ to $8.7 \pm 2.7 \mu \mathrm{g} / \mathrm{dL}(P=0.0053)$ during the morning infusion and $7.5 \pm 1.5$ to $5.6 \pm 2.2 \mathrm{~g} / \mathrm{dL}(P=0.0025)$ during the afternoon infusion, consistent with physiologic diurnal variation in endogenous cortisol. Area under the curve for serum cortisol from baseline to end of the afternoon infusion was higher with the ACTH infusion as compared with placebo infusion $(11,240 \pm 1369 \mu \mathrm{g} / \mathrm{dL}$ min vs $2992 \pm 579 \mu \mathrm{g} / \mathrm{dL}$ min, $P<0.0001)$. Additionally, 24-h urine collections that began in the morning of the infusions were assessed for urine-free cortisol (UFC). As anticipated, UFC values were higher when participants received cosyntropin as compared to placebo $(688.8 \pm 193.1$ vs $43.0 \pm 17.5 \mu \mathrm{g} /$ total volume, $P<0.0001)$.

\section{Impact of cosyntropin infusion on sclerostin}

Baseline serum sclerostin values were 362.7 (289.0-496.9) $\mathrm{pg} / \mathrm{mL}$ at the cosyntropin visit and 393.9 (279.6-576.7) $\mathrm{pg} / \mathrm{mL}$ at the placebo visit $(P=0.16)$. Using a mixed model analysis, controlled for fixed effects of age, sex, and BMI, we showed that there was no significant effect of cosyntropin as compared to placebo infusions on serum sclerostin levels at the end of the infusions and at $24 \mathrm{~h}(P=0.10)$ (Fig. 3). 


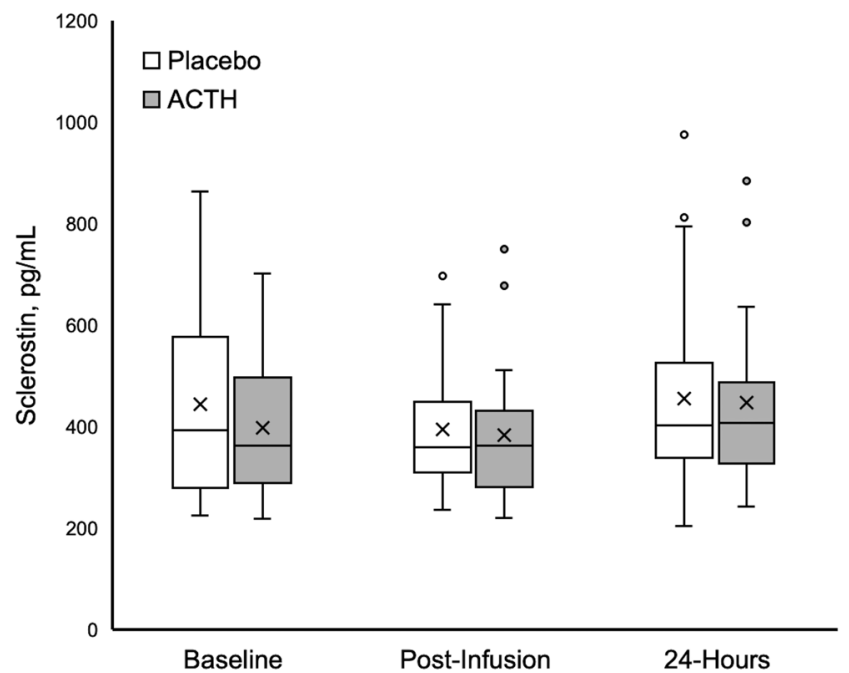

Figure 3

Effect of ACTH and placebo infusion on sclerostin concentrations. Serum sclerostin measurements were taken at baseline (pre-infusion), at the end of the afternoon infusion (approximately $6.5 \mathrm{~h}$ after the start of the morning infusion), and the following day ( $24 \mathrm{~h}$ after baseline measures). Placebo visit is represented by the white boxes and ACTH visit by the gray boxes. Whiskers extend to maximum and minimum value, the box represents the interquartile range, $X$ denotes the mean, and the line inside the box shows the median. Data points outside the whiskers show the outliers. A mixed model analysis showed no effect of cosyntropin vs placebo over time $(P=0.10)$.

We also examined the change in sclerostin from baseline to $24 \mathrm{~h}$ after baseline (approximately $16 \mathrm{~h}$ after the second infusion). The 24 -h change in sclerostin with cosyntropin as compared to placebo approached, but did not meet, statistical significance when analyzing men $(P=0.0867)$ and women $(P=0.0699)$ separately using a Fisher's exact test (Fig. 4), with a trend toward increased sclerostin. Twenty-four-hour UFC did not predict the sclerostin levels obtained the next day (after study infusions) for either the placebo or cosyntropin visits. UFC also did not predict the 24-h change in sclerostin from baseline to next day for either placebo or cosyntropin visits.

\section{Discussion}

This is the first placebo-controlled, randomized trial investigating the acute effects of an infusion of cosyntropin compared to placebo on sclerostin levels in healthy men and women. At baseline, there was a wide range in sclerostin values between participants, with women showing significantly lower values than men. However, there was notable intraindividual consistency in sclerostin levels over the 1-3 months between the study visits. Cosyntropin increased serum cortisol as expected;

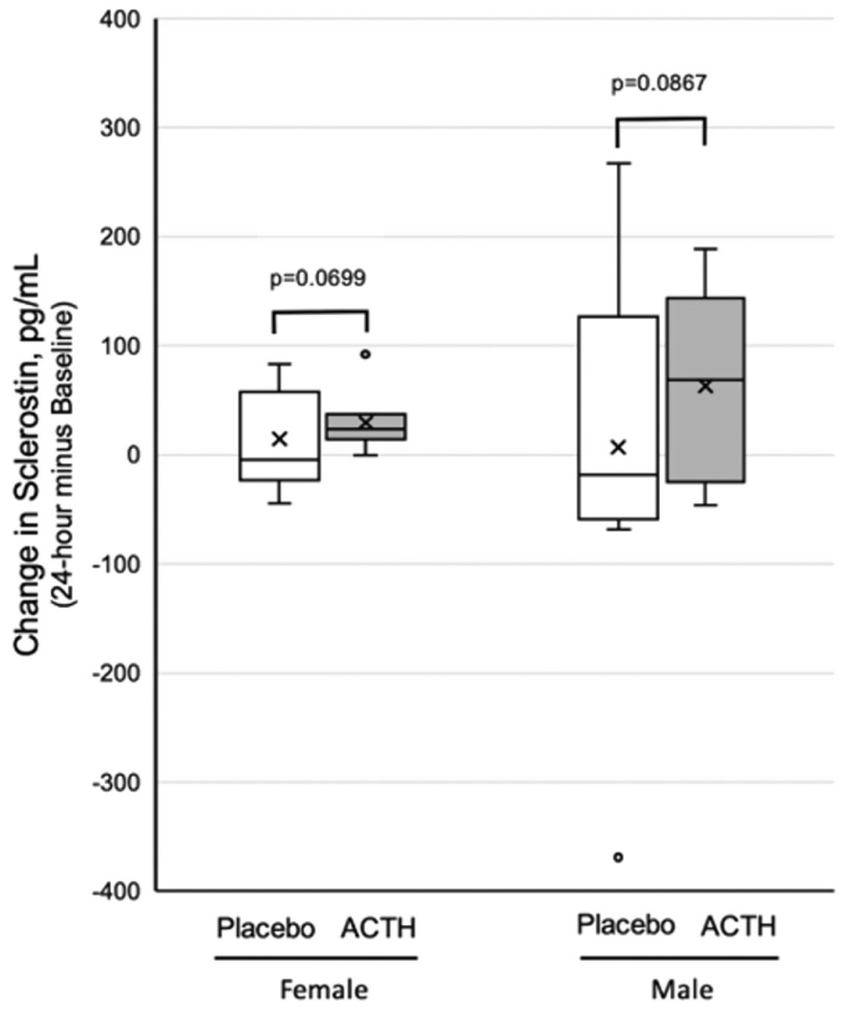

Figure 4

Sclerostin 24-h change from baseline in females $(n=7)$ and males $(n=10)$ after placebo or ACTH infusion. Twenty-four-hour change from baseline in serum sclerostin for the placebo visit is represented by the white boxes and ACTH visit by the gray boxes. Females are shown on the left and males on the right. Whiskers extend to maximum and minimum value, the box represents the interquartile range, $X$ denotes the mean, and the line inside the box shows the median. Data points outside the whiskers show the outliers. $P$ values were obtained using a Fisher's exact test for men and women separately.

we found no significant effect on serum sclerostin levels compared to placebo.

The interactions between the pituitary-adrenal axis and sclerostin are clinically important and poorly understood. Sclerostin is implicated in glucocorticoidinduced osteoporosis, and in animals, administration of glucocortoids increases sclerostin $(14,15)$. Observational studies in humans, both cross-sectional and prospective, have examined the impact of steroid use on sclerostin levels in individuals with underlying medical conditions with conflicting results. Serum sclerostin levels were shown to be significantly increased after 12 months of glucocorticoid therapy (mean dose of $64 \pm 16 \mathrm{mg} /$ day of prednisone) in patients with hematological disorders as compared to a control group of healthy individuals not receiving steroids (23). In contrast, glucocorticoid therapy was shown to decrease serum sclerostin as compared to baseline in patients with rheumatoid arthritis, polymyalgia 
rheumatica, and in those with a variety of medical illnesses $(24,25,27)$. The effect on sclerostin may vary with the duration of glucocorticoid therapy. For example, the initiation of glucocorticoid therapy (30-60 mg/day of prednisolone) in patients with systemic autoimmune diseases led to significant increases in serum sclerostin compared to baseline after 1 week of treatment, followed by significant decreases compared to baseline after 3-4 weeks of treatment (26). The sclerostin profile changes induced by glucocorticoids may also depend on the underlying medical condition. For example, the decrease in sclerostin levels seen in inflammatory conditions may be dependent on the glucocorticoid-related suppression of inflammation (27).

Additionally, there are studies examining sclerostin levels in individuals with endogenous hypercortisolism. Individuals with Cushing's syndrome have been shown to have higher serum sclerostin levels than healthy individuals (22). However, another study showed that individuals with endogenous hypercortisolism had significantly lower levels of plasma sclerostin when compared to controls (20), and the severity of cortisol excess has been negatively associated with sclerostin levels in patients with Cushing's syndrome (21). The variability in these findings may be due to the crosssectional and observational nature of these studies, the magnitude and duration of cortisol exposure, and additional underlying medical conditions. In healthy men studied on an inpatient clinical research center, blood sclerostin levels assessed every $2 \mathrm{~h}$ for $24 \mathrm{~h}$ were relatively stable with no significant diurnal rhythm (33). However, intense exercise has been shown to rapidly increase sclerostin in humans with one study showing a return to baseline levels $1 \mathrm{~h}$ after exercise completion (31). In contrast, sclerostin levels were chronically elevated for at least 30 days after zoledronic acid administration (34). Finally, an 18-h infusion of parathyroid hormone was shown to decrease sclerostin during the infusion in humans $(34,35)$. In mice, the half-life of sclerostin appears to be short, less than 5 min after intravenous administration (36). Preclinical studies have shown that changes in Sost gene transcription lead to changes in sclerostin protein in response to parathyroid hormone and mechanical loading (37). In addition, exposure to bone anabolic stimuli leads to a rapid, minute-scale increase in the degradation of sclerostin by lysosomes (38). Together, these studies suggest that the production and metabolism of sclerostin may vary depending on the biological conditions. The current study showed that infusion of ACTH did not lead to changes in sclerostin, despite increases in cortisol similar to those observed with intense exercise. The current study controlled for mechanical loading, a strong predictor of sclerostin, by dictating posture and activity levels during the course of each study visit. The rigorous control of conditions in our study is an important advantage, and likely contributed to the marked consistency in the baseline measurements across individuals.

Our study is the first of its kind to examine the acute effects of ACTH-induced rises in endogenous cortisol on serum sclerostin levels in healthy men and women. Consistent with other studies, we found that men have a significantly higher baseline sclerostin than women $(39,40,41)$. We were unable to demonstrate a positive or negative effect of cosyntropin on serum sclerostin levels. It is difficult to prove a negative effect. However, power calculations using our current data, which has a large S.D. likely due to our small sample size, suggests that we would need to study over 9000 individuals to achieve $80 \%$ power with an alpha of 0.05 and two tails hypothesis. In designing future studies, it will be important to account for the marked difference in baseline sclerostin between women and men. To this point, one of the main limitations of our study is the small sample size.

Another limitation relates to our method of inducing hypercortisolism. We used cosyntropin to stimulate adrenal cortisol production, which induces a relatively short period of hypercortisolemia, and it is possible that a longer duration of hypercortisolemia may be required to demonstrate a measureable effect on sclerostin levels. Additionally, changes in sclerostin could have occurred earlier than $6 \mathrm{~h}$, or after the 24-h observation period. Earlier and later time points should be assessed.

In healthy and relatively young men and women, we were unable to detect a significant change in sclerostin levels over a 24 -h period after infusion of cosyntropin as compared to placebo. It is reassuring for bone health that we did not see an acute rise in sclerostin with an ACTH infusion, as a positive finding would raise concerns over the impact of acute pituitary-adrenal axis activation on skeletal health.

\section{Declaration of interest}

The authors declare that there is no conflict of interest that could be perceived as prejudicing the impartiality of the research reported.

\section{Funding}

Gail Adler received support from the National Institutes of Health (K24HL103845); Janet Leung received support from National Institutes

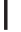

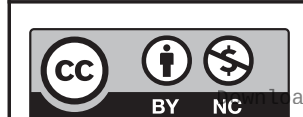


of Health (T32HL007609). This work was conducted with support from Harvard Catalyst | The Harvard Clinical and Translational Science Center (National Center for Advancing Translational Sciences, National Institutes of Health Award UL1 TR001102) and financial contributions from Harvard University and its affiliated academic healthcare centers. The content is solely the responsibility of the authors and does not necessarily represent the official views of Harvard Catalyst, Harvard University and its affiliated academic healthcare centers, or the National Institutes of Health.

\section{Author contribution statement}

Study design: J L and G K A. Study conduct: O B and J L. Data analysis and interpretation: $\mathrm{S} \mathrm{Z}, \mathrm{KM}, \mathrm{R} \mathrm{E}, \mathrm{O} \mathrm{B}$, and $\mathrm{M} \mathrm{H}$. Drafting manuscript: $\mathrm{S} Z$, $K M, R E$, and $O B$. Revising manuscript content: J $L, A K, M H, R F$, and G K A. Approving final manuscript: $S Z, K M, R E, O B, J L, A K, M H, R F$, and $G K A$. G K A takes responsibility for the integrity of the data and the accuracy of the data analysis.

\section{References}

1 Guañabens N, Gifre L \& Peris P. The role of Wnt signaling and sclerostin in the pathogenesis of glucocorticoid-induced osteoporosis. Current Osteoporosis Reports 201412 90-97. (https://doi.org/10.1007/ s11914-014-0197-0)

2 Baron R \& Kneissel M. WNT signaling in bone homeostasis and disease: from human mutations to treatments. Nature Medicine 2013 19 179-192. (https://doi.org/10.1038/nm.3074)

3 Rossini M, Gatti D \& Adami S. Involvement of WNT/ $\beta$-catenin signaling in the treatment of osteoporosis. Calcified Tissue International 201393 121-132. (https://doi.org/10.1007/s00223-0139749-z)

4 Ramli FF \& Chin KY. A review of the potential application of osteocyterelated biomarkers, fibroblast growth factor-23, sclerostin, and Dickkopf-1 in predicting osteoporosis and fractures. Diagnostics 2020 10 145. (https://doi.org/10.3390/diagnostics10030145)

5 Lin C, Jiang X, Dai Z, Guo X, Weng T, Wang J, Li Y, Feng G, Gao X \& He L. Sclerostin mediates bone response to mechanical unloading through antagonizing Wnt/beta-catenin signaling. Journal of Bone and Mineral Research 200924 1651-1661. (https://doi.org/10.1359/ jbmr.090411)

6 Poole KE, van Bezooijen RL, Loveridge N, Hamersma H, Papapoulos SE, Löwik CW \& Reeve J. Sclerostin is a delayed secreted product of osteocytes that inhibits bone formation. FASEB Journal 200519 1842-1844. (https://doi.org/10.1096/fj.05-4221fje)

7 Li X, Zhang Y, Kang H, Liu W, Liu P, Zhang J, Harris SE \& Wu D. Sclerostin binds to LRP5/6 and antagonizes canonical Wnt signaling. Journal of Biological Chemistry 2005280 19883-19887. (https://doi. org/10.1074/jbc.M413274200)

8 Winkler DG, Sutherland MK, Geoghegan JC, Yu C, Hayes T, Skonier JE, Shpektor D, Jonas M, Kovacevich BR, Staehling-Hampton K, et al. Osteocyte control of bone formation via sclerostin, a novel BMP antagonist. EMBO Journal 200322 6267-6276. (https://doi org/10.1093/emboj/cdg599)

9 Ohnaka K, Tanabe M, Kawate H, Nawata H \& Takayanagi R. Glucocorticoid suppresses the canonical Wnt signal in cultured human osteoblasts. Biochemical and Biophysical Research Communications 2005329 177-181. (https://doi.org/10.1016/j. bbrc.2005.01.117)

10 Compston J. Glucocorticoid-induced osteoporosis: an update. Endocrine 201861 7-16. (https://doi.org/10.1007/s12020-018-1588-2)

11 Henneicke H, Gasparini SJ, Brennan-Speranza TC, Zhou H \& Seibel MJ. Glucocorticoids and bone: local effects and systemic implications. Trends in Endocrinology and Metabolism 201425 197-211. (https://doi. org/10.1016/j.tem.2013.12.006)
12 O'Brien CA, Jia D, Plotkin LI, Bellido T, Powers CC, Stewart SA, Manolagas SC \& Weinstein RS. Glucocorticoids act directly on osteoblasts and osteocytes to induce their apoptosis and reduce bone formation and strength. Endocrinology 2004145 1835-1841. (https:// doi.org/10.1210/en.2003-0990)

13 Weinstein RS, Jilka RL, Parfitt AM \& Manolagas SC. Inhibition of osteoblastogenesis and promotion of apoptosis of osteoblasts and osteocytes by glucocorticoids. Potential mechanisms of their deleterious effects on bone. Journal of Clinical Investigation $1998 \mathbf{1 0 2}$ 274-282. (https://doi.org/10.1172/JCI2799)

14 Yao W, Cheng Z, Busse C, Pham A, Nakamura MC \& Lane NE. Glucocorticoid excess in mice results in early activation of osteoclastogenesis and adipogenesis and prolonged suppression of osteogenesis: a longitudinal study of gene expression in bone tissue from glucocorticoid-treated mice. Arthritis and Rheumatism $2008 \mathbf{5 8}$ 1674-1686. (https://doi.org/10.1002/art.23454)

15 Beier EE, Sheu TJ, Resseguie EA, Takahata M, Awad HA, CorySlechta DA \& Puzas JE. Sclerostin activity plays a key role in the negative effect of glucocorticoid signaling on osteoblast function in mice. Bone Research 20175 17013. (https://doi.org/10.1038/ boneres.2017.13)

16 Marenzana M, Greenslade K, Eddleston A, Okoye R, Marshall D, Moore A \& Robinson MK. Sclerostin antibody treatment enhances bone strength but does not prevent growth retardation in young mice treated with dexamethasone. Arthritis and Rheumatism 201163 2385-2395. (https://doi.org/10.1002/art.30385)

17 Yao W, Dai W, Jiang L, Lay EY, Zhong Z, Ritchie RO, Li X, Ke H \& Lane NE. Sclerostin-antibody treatment of glucocorticoid-induced osteoporosis maintained bone mass and strength. Osteoporosis International 201627 283-294. (https://doi.org/10.1007/s00198-0153308-6)

18 Li X, Ominsky MS, Niu QT, Sun N, Daugherty B, D'Agostin D, Kurahara C, Gao Y, Cao J, Gong J, et al. Targeted deletion of the sclerostin gene in mice results in increased bone formation and bone strength. Journal of Bone and Mineral Research 200823 860-869. (https://doi.org/10.1359/jbmr.080216)

19 Sato AY, Cregor M, Delgado-Calle J, Condon KW, Allen MR, Peacock M, Plotkin LI \& Bellido T. Protection from glucocorticoid-induced osteoporosis by anti-catabolic signaling in the absence of Sost/ sclerostin. Journal of Bone and Mineral Research 201631 1791-1802. (https://doi.org/10.1002/jbmr.2869)

20 van Lierop AH, van der Eerden AW, Hamdy NA, Hermus AR, den Heijer M \& Papapoulos SE. Circulating sclerostin levels are decreased in patients with endogenous hypercortisolism and increase after treatment. Journal of Clinical Endocrinology and Metabolism 201297 E1953-E1957. (https://doi.org/10.1210/jc.2012-2218)

21 Athimulam S, Delivanis D, Thomas M, Young WF, Khosla S, Drake MT $\&$ Bancos I. The impact of mild autonomous cortisol secretion on bone turnover markers. Journal of Clinical Endocrinology and Metabolism 2020105 1469-1477. (https://doi.org/10.1210/clinem/dgaa120)

22 Belaya ZE, Rozhinskaya LY, Melnichenko GA, Solodovnikov AG, Dragunova NV, Iljin AV, Dzeranova LK \& Dedov II. Serum extracellular secreted antagonists of the canonical Wnt/ $\beta$-catenin signaling pathway in patients with Cushing's syndrome. Osteoporosis International 201324 2191-2199. (https://doi.org/10.1007/s00198-0132268-y)

23 Gifre L, Ruiz-Gaspà S, Monegal A, Nomdedeu B, Filella X, Guañabens N \& Peris P. Effect of glucocorticoid treatment on Wnt signalling antagonists (sclerostin and Dkk-1) and their relationship with bone turnover. Bone 201357 272-276. (https://doi.org/10.1016/j. bone.2013.08.016)

24 Brabnikova Maresova K, Pavelka K \& Stepan JJ. Acute effects of glucocorticoids on serum markers of osteoclasts, osteoblasts, and osteocytes. Calcified Tissue International 201392 354-361. (https://doi. org/10.1007/s00223-012-9684-4) https://ec.bioscientifica.com https://doi.org/10.1530/EC-21-0263 (c) 2021 The authors Published by Bioscientifica Ltd

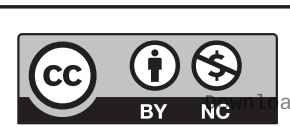

This work is licensed under a Creative Commons Attribution-NonCommercial 4.0 International License. ded from Bioscientifica.com at 04/26/2023 04:54:19AM 
25 Fassio A, Adami G, Giollo A, Viapiana O, Malavolta N, Saviola G, Bortolotti R, Idolazzi L, Bertoldo F, Rossini M, et al. Acute effects of glucocorticoid treatment, TNF $\alpha$ or IL-6R blockade on bone turnover markers and Wnt inhibitors in early rheumatoid arthritis: a pilot study. Calcified Tissue International 2020106 371-377. (https://doi. org/10.1007/s00223-019-00649-3)

26 Kawazoe M, Kaneko K, Shikano K, Kusunoki N, Nanki T \& Kawai S. Glucocorticoid therapy causes contradictory changes of serum Wnt signaling-related molecules in systemic autoimmune diseases. Clinical Rheumatology 201837 2169-2178. (https://doi.org/10.1007/s10067-0173689-3)

27 Fassio A, Adami G, Idolazzi L, Giollo A, Viapiana O, Vantaggiato E, Benini C, Rossini M, Dejaco C \& Gatti D. Wnt inhibitors and bone turnover markers in patients with polymyalgia rheumatica and acute effects of glucocorticoid treatment. Frontiers in Medicine 20207551. (https://doi.org/10.3389/fmed.2020.00551)

28 Jacobsson M, van Raalte DH, Heijboer AC, den Heijer M \& de Jongh RT. Short-term glucocorticoid treatment reduces circulating sclerostin concentrations in healthy young men: a randomized, placebocontrolled, double-blind study. JBMR Plus 20204 e10341. (https://doi. org/10.1002/jbm4.10341)

29 Pickering ME, Simon M, Sornay-Rendu E, Chikh K, Carlier MC, Raby AL, Szulc P \& Confavreux CB. Serum sclerostin increases after acute physical activity. Calcified Tissue International 2017101 170-173. (https://doi.org/10.1007/s00223-017-0272-5)

30 Kouvelioti R, Kurgan N, Falk B, Ward WE, Josse AR \& Klentrou P. Response of sclerostin and bone turnover markers to high intensity interval exercise in young women: does impact matter? BioMed Research International 20182018 4864952. (https://doi. org/10.1155/2018/4864952)

31 Diaz-Castro J, Mira-Rufino PJ, Moreno-Fernandez J, Chirosa I, Chirosa JL, Guisado R \& Ochoa JJ. Ubiquinol supplementation modulates energy metabolism and bone turnover during high intensity exercise. Food and Function 202011 7523-7531. (https://doi. org/10.1039/dOfo01147a)

32 Leung JH, Bayomy OF, Bonyhay I, Celli J, White J, Freeman R \& Adler GK. ACTH infusion impairs baroreflex sensitivity-implications for cardiovascular hypoglycemia-associated autonomic failure. Journal of Clinical Endocrinology and Metabolism 2020105 2345-2352. (https:// doi.org/10.1210/clinem/dgaa221)
33 Swanson C, Shea SA, Wolfe P, Markwardt S, Cain SW, Munch M, Czeisler CA, Orwoll ES \& Buxton OM. 24-hour profile of serum sclerostin and its association with bone biomarkers in men. Osteoporosis International 201728 3205-3213. (https://doi.org/10.1007/ s00198-017-4162-5)

34 Catalano A, Morabito N, Basile G, Brancatelli S, Cucinotta D \& Lasco A. Zoledronic acid acutely increases sclerostin serum levels in women with postmenopausal osteoporosis. Journal of Clinical Endocrinology and Metabolism 201398 1911-1915. (https://doi.org/10.1210/jc.2012-4039)

35 Yu EW, Kumbhani R, Siwila-Sackman E \& Leder BZ. Acute decline in serum sclerostin in response to PTH infusion in healthy men. Journal of Clinical Endocrinology and Metabolism 201196 E1848-E1851. (https:// doi.org/10.1210/jc.2011-1534)

36 Dreyer T, Shah M, Doyle C, Greenslade K, Penney M, Creeke P, Kotian A, Ke HZ, Naidoo V \& Holdsworth G. Recombinant sclerostin inhibits bone formation in vitro and in a mouse model of sclerosteosis. Journal of Orthopaedic Translation 202129 134-142. (https://doi. org/10.1016/j.jot.2021.05.005)

37 Sebastian A \& Loots GG. Transcriptional control of Sost in bone. Bone 201796 76-84. (https://doi.org/10.1016/j.bone.2016.10.009)

38 Gould NR, Williams KM, Joca HC, Torre OM, Lyons JS, Leser JM, Srikanth MP, Hughes M, Khairallah RJ, Feldman RA, et al. Disparate bone anabolic cues activate bone formation by regulating the rapid lysosomal degradation of sclerostin protein. eLife $2021 \mathbf{1 0}$ e64393. (https://doi.org/10.7554/eLife.64393)

39 Amrein K, Amrein S, Drexler C, Dimai HP, Dobnig H, Pfeifer K, Tomaschitz A, Pieber TR \& Fahrleitner-Pammer A. Sclerostin and its association with physical activity, age, gender, body composition, and bone mineral content in healthy adults. Journal of Clinical Endocrinology and Metabolism 201297 148-154. (https://doi. org/10.1210/jc.2011-2152)

40 Xu Y, Gao C, He J, Gu W, Yi C, Chen B, Wang Q, Tang F, Xu J, Yue H, et al. Sclerostin and its associations with bone metabolism markers and sex hormones in healthy community-dwelling elderly individuals and adolescents. Frontiers in Cell and Developmental Biology 2020857. (https://doi.org/10.3389/fcell.2020.00057)

41 Mödder UI, Hoey KA, Amin S, McCready LK, Achenbach SJ, Riggs BL, Melton LJ \& Khosla S. Relation of age, gender, and bone mass to circulating sclerostin levels in women and men. Journal of Bone and Mineral Research 201126 373-379. (https://doi.org/10.1002/jbmr.217)

Received in final form 12 October 2021

Accepted 17 November 2021

Accepted Manuscript published online 17 November 2021 https://ec.bioscientifica.com https://doi.org/10.1530/EC-21-0263
(C) 2021 The authors Published by Bioscientifica Ltd
This work is licensed under a Creative Commons Attribution-NonCommercial 4.0 International License. ded from Bioscientifica.com at 04/26/2023 04:54:19AM 